\title{
First evidence of low energy enhancement in Ge isotopes
}

T. Renstrøm ${ }^{1, a}$, H.-T. Nyhus ${ }^{1}$, H. Utsunomiya ${ }^{2}$, A. C. Larsen ${ }^{1}$, S. Siem ${ }^{1}$, M. Guttormsen ${ }^{1}$, D. M. Filipescu ${ }^{3,4}$, I. Gheorghe ${ }^{3,4,5}$, S. Goriely ${ }^{6}$, L. A. Bernstein ${ }^{7}$, D. L. Bleuel ${ }^{7}$, T. Glodariu ${ }^{4}$, A. Görgen ${ }^{1}$, T. W. Hagen ${ }^{1}$, Y.-W. Lui ${ }^{8}$, D. Negi ${ }^{9}$, I. E. Ruud ${ }^{1}$, E. Şahin ${ }^{1}$, R. Schwengner ${ }^{10}$, T. Shima ${ }^{11}$, K. Takahisa ${ }^{11}$, O. Tesileanu ${ }^{3}$, T. G. Tornyi ${ }^{12}$, G. M. Tveten ${ }^{1}$, and M. Wiedeking ${ }^{9}$

${ }^{1}$ Department of Physics, University of Oslo, N-0316 Oslo, Norway

${ }^{2}$ Department of Physics, Konan University, Okamoto 8-9-1, Higashinada, Kobe 658-8501, Japan

${ }^{3}$ Extreme Light Infrastructure Nuclear Physics, str Atomistilor nr. 407, Bucharest-Magurele, P.O.BOX MG6, Romania

${ }^{4}$ National Institute for Physics and Nuclear Engineering Horia Hulubei, str Atomistilor nr. 407, Bucharest-Magurele, P.O.BOX MG6, Romania

${ }^{5}$ Faculty of Physics, University of Bucharest, Bucharest, Romania

${ }^{6}$ Institut d'Astronomie et d'Astrophysique, ULB, Belgium

${ }^{7}$ Lawrence Livermore National Laboratory, 7000 East Avenue, Livermore, California 94550-9234, US

${ }^{8}$ Cyclotron Institute, Texas A\&M University, College Station, Texas 77843, USA

${ }^{9}$ iThemba LABS, P.O. Box 722, 7129 Somerset West, South Africa

${ }^{10}$ Institute of Radiation Physics, Helmholtz-Zentrum Dresden-Rossendorf, 01328 Dresden, Germany

${ }^{11}$ Research Center for Nuclear Physics, Osaka University, Suita, Osaka 567-0047, Japan

${ }^{12}$ Institute of Nuclear Research of the Hungarian Academy of Sciences (MTA Atomki), Debrecen, Hungary

\begin{abstract}
.
The $\gamma$-strength functions and level densities of ${ }^{73,74} \mathrm{Ge}$ have been extracted from particle- $\gamma$ coincidence data using the Oslo method. In addition the $\gamma$-strength function of ${ }^{74} \mathrm{Ge}$ above the neutron separation threshold, $\mathrm{S}_{n}$ $=10.196 \mathrm{MeV}$ has been extracted from photoneutron measurements. When combined, these two experiments give a $\gamma$-strength function covering the energy range of $\sim 1-13 \mathrm{MeV}$ for ${ }^{74} \mathrm{Ge}$. This thorough investigation of ${ }^{74} \mathrm{Ge}$ is a part of an international campaign to study the previously reported low energy enhancement in this mass region in the $\gamma$-strength function from $\sim 3 \mathrm{MeV}$ towards lower $\gamma$ energies. The obtained data show that both ${ }^{73,74} \mathrm{Ge}$ display an increase in strength at low $\gamma$ energies.
\end{abstract}

\section{Introduction}

The $\gamma$-ray strength function [1] has been proven to be a useful concept to characterize the average nuclear response to electromagnetic radiation when the nucleus is excited to high energies, and the density of quantum levels is high. There exists a wealth of information about the $\gamma$-strength function $(\gamma \mathrm{SF})$ for nuclei above the neutron binding energy, predominantly from photoneutron experiments [2]. For $\gamma$ energies below $\mathrm{S}_{n}$ the information is more scarce, as the strength is quite challenging to extract in this area. Methods such as the Oslo method [3], the two-step cascade method [4] and a statistical treatment of nuclearresonance fluorescence spectra [5] are used to investigate the strength below the neutron separation energy. However, the results from the different techniques sometimes show large discrepancies in the resulting $\gamma \mathrm{SF}$. For energies below $\sim 3 \mathrm{MeV}$, the $\gamma \mathrm{SF}$ of a nucleus is dominated by the receding tail of the giant electric dipole resonance. It therefore came as a surprise when a strong low-energy enhancement in the $\gamma \mathrm{SF}$, hereafter referred to as the up- bend, was discovered below $3 \mathrm{MeV}$ for ${ }^{56,57} \mathrm{Fe}$ [6]. The $\gamma \mathrm{SF}$ measurement was performed at Oslo Cyclotron Laboratory (OCL), using charged particle reactions (and confirmed using the two-step cascade method), and the following years this phenomenon was observed in a wide range of nuclei [7-13]. A set of examples of upbends are shown in Fig.1. Recently, the upbend was reported with a different experimental technique in ${ }^{95} \mathrm{Mo}$ [14]. The physical mechanisms behind the upbend was a puzzle for many years, but recently three papers with theoretical explanations have been published [15-17]. In addition, the upbend has been experimentally shown to be dominantly of dipole nature [18]. An international collaboration was formed with the common goal of investigating one specific nucleus in the mass range where the upbend structure was likely to appear, using different experimental techniques. The nucleus ${ }^{74} \mathrm{Ge}$ was chosen, and four different experiments were performed: $\left({ }^{3} \mathrm{He},{ }^{3} \mathrm{He}^{\prime}\right),\left(\alpha, \alpha^{\prime}\right),\left(\mathrm{p}, \mathrm{p}^{\prime}\right)$ and $\left(\gamma, \gamma^{\prime}\right)$. In the following we will present our results from ${ }^{3} \mathrm{He}$ induced reactions on ${ }^{74} \mathrm{Ge}$ performed at OCL, and furthermore, we will show new results from a photoneutron

\footnotetext{
ae-mail: therese.renstrom@fys.uio.no
} 


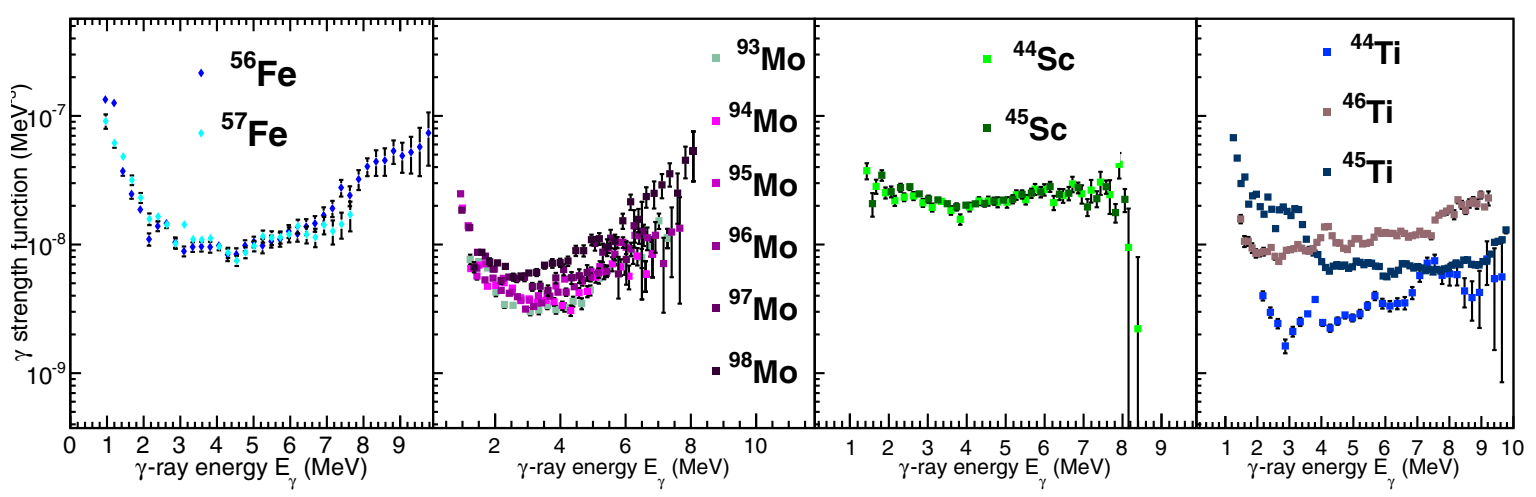

Figure 1. Some examples of low energy enhancements in the $\gamma$-ray strength function found in medium mass nuclei, using the Oslo method.

experiment on ${ }^{74} \mathrm{Ge}$ performed at the NewSUBARU laboratory in Japan.

\section{Experimental methods}

We used two different approaches to investigate the $\gamma \mathrm{SF}$ of ${ }^{74} \mathrm{Ge}$, a charged particle reaction and a photoneutron experiment. We will in the following sections present the two different techniques briefly.

\subsection{Oslo data}

The experiment was performed at the OCL, where $\mathrm{a} \sim 0.5$ enA ${ }^{3} \mathrm{He}$ beam at $38 \mathrm{MeV}$ bombarded a self-supporting, $0.5 \mathrm{mg} / \mathrm{cm}^{2}$ thick ${ }^{74} \mathrm{Ge}$ target. The two reaction channels of interest were ${ }^{74} \mathrm{Ge}\left({ }^{3} \mathrm{He},{ }^{3} \mathrm{He}^{\prime} \gamma\right)$ and ${ }^{74} \mathrm{Ge}\left({ }^{3} \mathrm{He}, \alpha \gamma\right)$. The charged outgoing particles were identified and their energies measured with the SiRi system [19], consisting of 64 $\Delta \mathrm{E}-\mathrm{E}$ silicon telescopes, with thicknesses of $130-\mu \mathrm{m}$ and $1550-\mu \mathrm{m}$, respectively. SiRi was placed in forward direction, covering angles from $\theta=40$ to $54^{\circ}$. The $\gamma$ rays were detected with the CACTUS array [20] consisting of $285^{\prime \prime} \times 5^{\prime \prime} \mathrm{NaI}(\mathrm{Tl})$ detectors surrounding the target and the particle detectors. The total efficiency of CACTUS is $15.2 \%$ at $\mathrm{E}_{\gamma}=1.3 \mathrm{keV}$. Using reaction kinematics, the initial excitation energy of the residual nucleus can be deduced from the energy of the outgoing particles detected in SiRi. The particle- $\gamma$ coincidence technique is used to assign each $\gamma$ ray to a cascade depopulating a certain initial excitation energy in the residual nucleus. Fig. 2(a) shows the particle- $\gamma$ matrix $\left(\mathrm{E}_{\gamma}, \mathrm{E}\right)$ of the ${ }^{74} \mathrm{Ge}\left({ }^{3} \mathrm{He},{ }^{3} \mathrm{He}^{\prime} \gamma\right)$ reaction, where the $\gamma$ spectra have been unfolded with the $\mathrm{NaI}$ response functions [21]. The neutron binding energy of ${ }^{74} \mathrm{Ge}$ is reflected clearly in a drop in $\gamma$ intensity at $\mathrm{E} \approx$ $\mathrm{S}_{n}=10.196 \mathrm{MeV}$. A quite weak diagonal, where $\mathrm{E}=\mathrm{E}_{\gamma}$ reveals that the direct feeding to the $0^{+}$ground state is not particularly favored. We see a second more pronounced diagonal that represents decay to the first excited $2^{+}$state of $596 \mathrm{keV}$. We know that these $\gamma$ rays stem from primary transitions in the $\gamma$ cascades, but the rest of the matrix consists of a mix of higher generation $\gamma$ rays. We would like to study the energy distribution of all primary $\gamma$-rays originating from various excitation energies, and extract the nuclear level density (NLD) and $\gamma \mathrm{SF}$ simultaneously from this information. From the unfolded $\gamma$ spectra, the distribution of primary $\gamma$ rays $P\left(E, E_{\gamma}\right)$, as shown in Fig. 2(b), has been extracted from the full cascades by the iterative subtraction method of Ref. [22].

In the quasi-continuum we assume that $P$ is proportional to the NLD at the final excitation energy $\rho\left(E-E_{\gamma}\right)$ and that the decay is governed by the $\gamma$-transmission coefficient $\mathcal{T}\left(E_{\gamma}\right)$, which according to the Brink hypothesis $[23,24]$ is independent of excitation energy. Thus the decay probability is given by

$$
P\left(E, E_{\gamma}\right) \propto \mathcal{T}\left(E_{\gamma}\right) \rho\left(E-E_{\gamma}\right) .
$$

The $\gamma \mathrm{SF}$ is related to the transmission coefficient by $f\left(E_{\gamma}\right)=\mathcal{T}\left(E_{\gamma}\right) / 2 \pi E_{\gamma}^{3}$, if we assume that statistical decay is dominated by dipole transitions. From Eq.(1) we see that the NLD and $\gamma \mathrm{SF}$ can be determined from a simultaneous fit to the primary $\gamma$ matrix, $P$.

\subsection{NewSUBARU data}

The photoneutron cross section measurements were performed at the synchrotron radiation facility NewSUBARU [25]. Quasi monochromatic $\gamma$-ray beams [26] are produced in head-on collisions between laser photons and relativistic electrons, a so-called laser-Compton scattering (LCS). The energy of the laser photons increases from a few eV to several MeV in the collisions. In this experiment a $1.9916 \mathrm{~g} / \mathrm{cm}^{2}$ thick sample of ${ }^{74} \mathrm{Ge}$, was placed inside an aluminum container and irrradiated with eight different $\gamma$-ray beams with energies from 10.4 to $12.7 \mathrm{MeV}$. The ${ }^{74} \mathrm{Ge}$ sample was mounted in the center of a $4 \pi$ neutron detection array comprised of $20{ }^{3} \mathrm{He}$ proportional counters embedded in a polyethylene moderator. The ring ratio technique [27] was used to measure the average energies of the detected neutrons, and from this the efficiency of the neutron detector is established. A $6^{\prime \prime} \times 5^{\prime \prime} \mathrm{NaI}(\mathrm{Tl})$ detector was used to measure the flux of the LCS beam. 


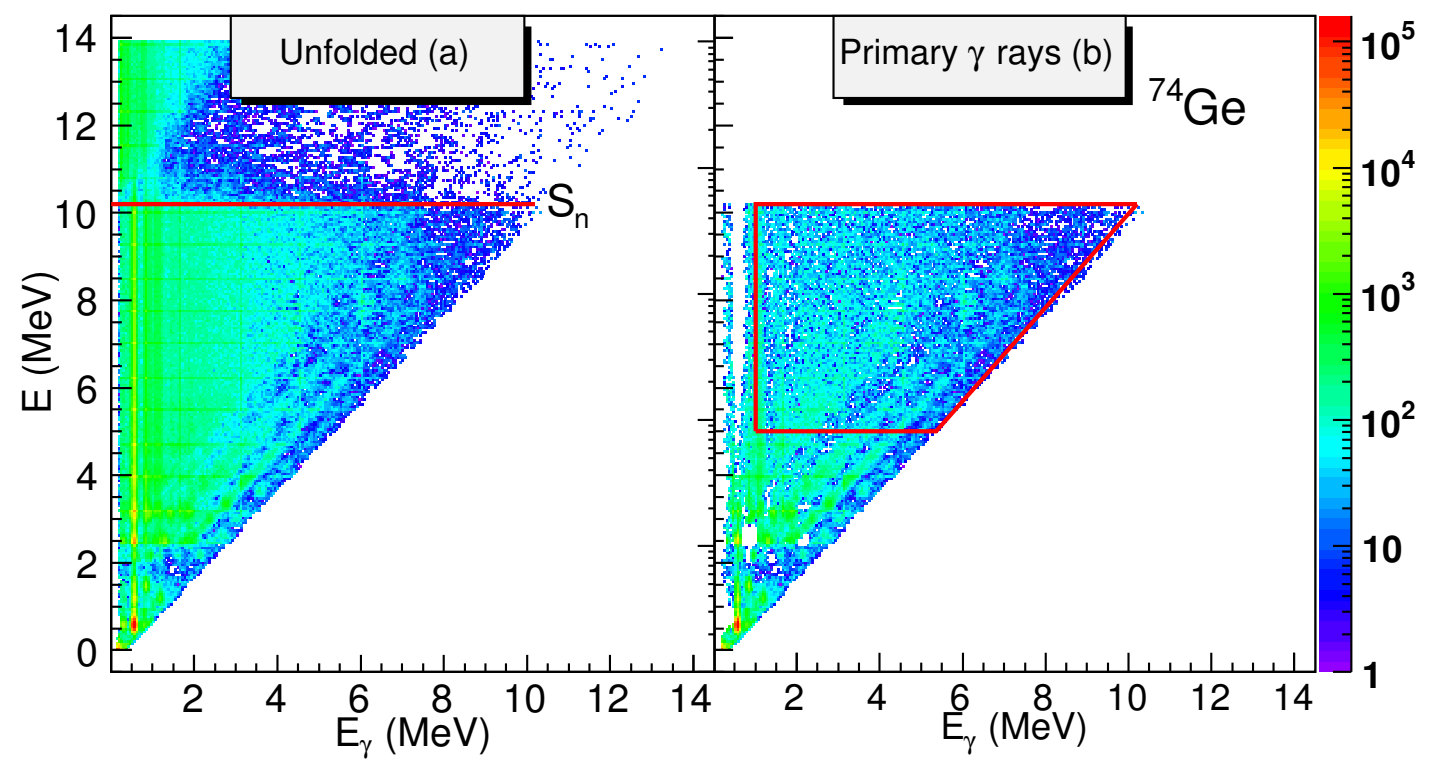

Figure 2. (a) Particle- $\gamma$ coincidence matrix from the ${ }^{74} \mathrm{Ge}\left({ }^{3} \mathrm{He},{ }^{3} \mathrm{He}^{\prime} \gamma\right){ }^{74} \mathrm{Ge}$ reaction. The NaI spectra are unfolded with the NaI response functions. (b) The first generation matrix.

The detector was placed at the end of the $\gamma$ ray beam line. The total number of $\gamma$ rays on target for a certain beam was found using the well established pile up method described in Ref. [28]. The almost monochromatic $\gamma$ beam was monitored by a $3.5^{\prime \prime} \times 4.0^{\prime \prime} \mathrm{LaBr}_{3}(\mathrm{Ce})$ detector. The spectra were reproduced using GEANT4 simulations, and unfolded to give the real energy profile of the incoming beam. The resolution of the $\gamma$-ray beams used in the experiment was excellent, typically $1-2 \%$ at FWHM.

The $(\gamma, \mathrm{n})$ cross section is given by,

$$
\int_{S_{n}}^{E_{\mathrm{Max}}} n_{\gamma}\left(E_{\gamma}\right) \sigma\left(E_{\gamma}\right) d E_{\gamma}=\frac{N_{n}}{N_{t} N_{\gamma} \xi \epsilon_{n} g},
$$

where $n_{\gamma}\left(E_{\gamma}\right)$ gives the energy distribution of the $\gamma$-ray beam normalized to unity and $\sigma\left(E_{\gamma}\right)$ is the photoneutron cross section to be determined. Furthermore, $N_{n}$ represents the number of neutrons detected, $N_{t}$ gives the number of target nuclei per unit area, $N_{\gamma}$ is the number of $\gamma$ rays incident on target, $\epsilon_{n}$ represents the neutron detection efficiency, and finally $\xi=\left(1-e^{\mu t}\right) /(\mu t)$ is the correction factor for a thick target measurement. The factor $g$ represents the fraction of $\gamma$ flux above the neutron threshold $S_{n}$. Eq.(2) is solved for the cross section using the Taylor expansion method described in [29]. In this way we find cross sections for eight different energies. The uncertainties in the measurements are $\sim 4.4 \%$ [29]. The measured photoneutron cross section are shown in Fig. 3 together with existing data [31]. We notice that our new data are on average $30 \%$ lower than the existing data. This is consisent with the (n, $\gamma$ ) cross sections measured for several $\mathrm{Nd}$ and $\mathrm{Sm}$ isotopes [29, 30] during the same experimental campaign at NewSUBARU.

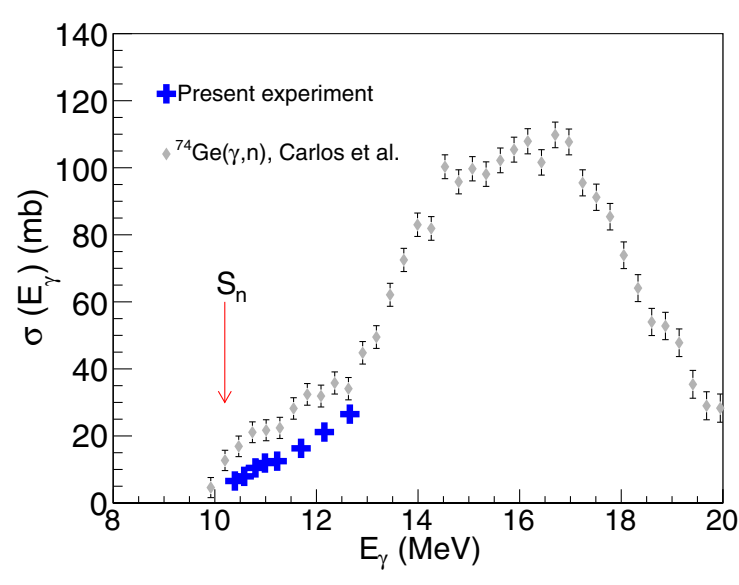

Figure 3. Comparison between present photoneutron emission cross sections for ${ }^{74} \mathrm{Ge}$ and previously measured ones [31]. The results are preliminary. by

The photoneutron cross sections are related to the $\gamma \mathrm{SF}$

$$
f\left(E_{\gamma}\right)=\frac{1}{3 \pi^{2} \hbar^{2} c^{2}} \frac{\sigma\left(E_{\gamma}\right)}{E_{\gamma}}
$$

which can be directly compared with the Oslo data from the principle of detailed balance, giving $f_{\text {up }} \approx f_{\text {down }}[1$, 32]. 


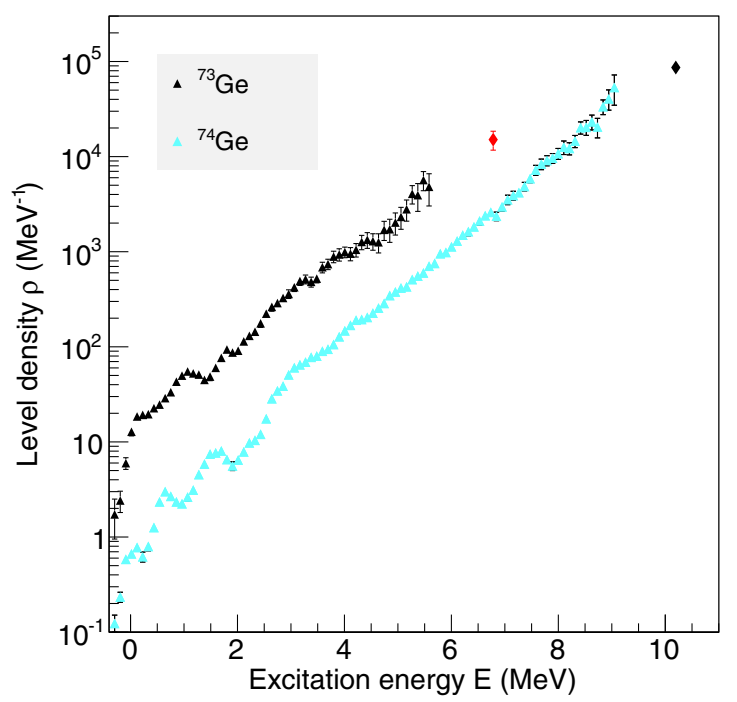

Figure 4. The NLDs of ${ }^{73,74} \mathrm{Ge}$. The red and black diamond shaped points represent the estimated NLD at the neutron binding energy of the two isotopes. The results are preliminary.

\section{Discussion of results}

After careful analysis of the photoneutron data and normalization of the Oslo data, we will briefly present at the NLDs, investigate if the two data set give us a consistent $\gamma \mathrm{SF}$ for ${ }^{74} \mathrm{Ge}$ in this broad energy range and observe the shapes of the $\gamma \mathrm{SFs}$.

The normalized NLDs of ${ }^{73,74} \mathrm{Ge}$ are shown in Fig. 4. We notice that ${ }^{74} \mathrm{Ge}$ has a constant temperature shape, ${ }^{73} \mathrm{Ge}$ has slightly more structures. We also observe that two isotopes have quite parallel NLD curves, as previously observed in the NLDs of many pairs of neighboring isotopes investigated with the Oslo method, see Ref. [33].

We now turn to the normalized $\gamma \mathrm{SFs}$, shown in Fig. 5. The two Ge isotopes are in very good agreement, as we previously have observed for several isotopic chains, see Fig. 1. There is also a nice correspondence in slope between the Oslo data and the new photoneutron data. Around $7 \mathrm{MeV}$ we see a distinct resonance-like structure in the strength of ${ }^{74} \mathrm{Ge}$. This structure has also been observed in the ${ }^{74} \mathrm{Ge}\left(\alpha, \alpha^{\prime}\right)$ data analyzed as a part of the Ge collaboration, and should be investigated further. We also observe that both ${ }^{73} \mathrm{Ge}$ and ${ }^{74} \mathrm{Ge}$ show a clear enhancement in the $\gamma \mathrm{SF}$ for gamma energies below $\sim 3 \mathrm{MeV}$. This means that the probability of $\gamma$ decay increases with decreasing $\gamma$ ray energy for both isotopes. The absolute value of the $\gamma \mathrm{SF}$ increases by a factor of 3 between $\sim 3 \mathrm{MeV}$ and 1 $\mathrm{MeV}$.

We know that the radiative neutron capture cross sections are sensitively depending on the $\gamma \mathrm{SF}$ around the neutron separation energy. The neutron threshold energy decreases for neutron rich nuclei, and if we assume that the upbend will persist when we move towards more neutron rich nuclei, approaching the neutron drip line this upbend

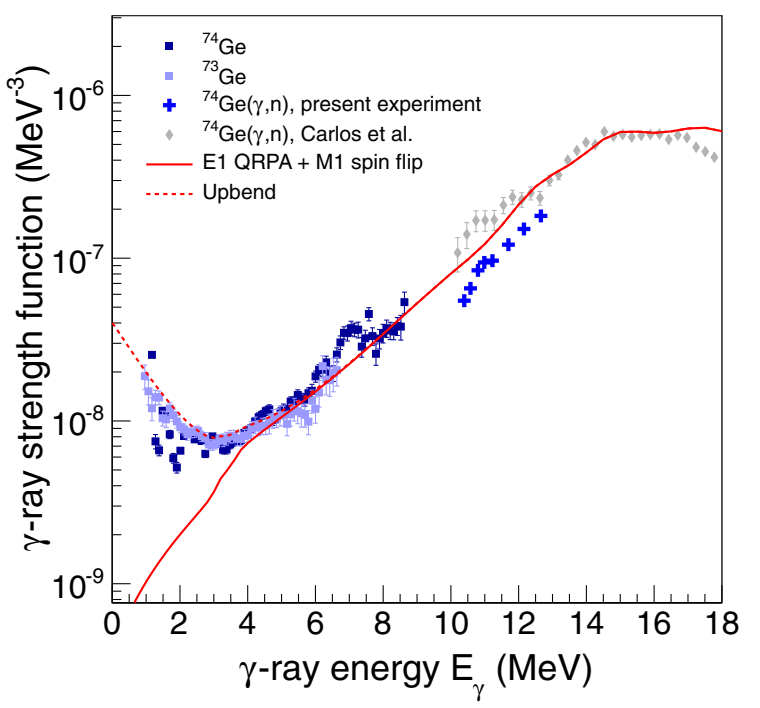

Figure 5. The $\gamma \mathrm{SFs}$ of ${ }^{73,74} \mathrm{Ge}$, including the $(\gamma, \mathrm{n})$ reaction for ${ }^{74} \mathrm{Ge}$ from the present experiment and from existing data [31]. The red solid line is the sum of the M1 spin flip resonance and the E1 strength calculated using QRPA calculations. The red dotted line includes in addition a parameterization of the upbend. The results are preliminary.

is likely to affect the $(\mathrm{n}, \gamma)$ reaction rates as previously shown in [34].

\section{Outlook}

We will continue our investigations by calculating the unknown ${ }^{73} \mathrm{Ge}(\mathrm{n}, \gamma){ }^{74} \mathrm{Ge}$ cross section and investigate the effect of the upbend on the reaction rates with and without upbend feature using TALYS-1.6 reaction code [35]. Also a careful analysis of the uncertainties connected to the normalization of the Oslo data will be performed.

Very recently a new and promising technique was developed at NSCL/MSU by Spyrou et al. [36] to measure NLD and $\gamma \mathrm{SF}$ in unstable neutron rich isotopes. The first results from the new method gave a strong constraint on the ${ }^{75} \mathrm{Ge}(\mathrm{n}, \gamma){ }^{76} \mathrm{Ge}$ reaction rate, not accessible directly because of the radioactive nature of ${ }^{75} \mathrm{Ge}$. We notice that the $\gamma \mathrm{SF}$ extracted for ${ }^{76} \mathrm{Ge}$ also shows a low energy enhancement. Proposals to measure neutron-rich Ge isotopes at MSU are in preparation.

\section{References}

[1] G. A. Bartholomew, E. D. Earle, A. J. Fergusson, J. W. Knowles, and A. J. Fergusson, Adv. Nucl. Phys. 7, $229(1972 / 73)$

[2] S. S. Dietrich and B. L. Berman, At. Data Nucl. Data Tables 38, 199 (1988)

[3] A. Schiller et al., Nucl. Instrum. Methods Phys. Res. A 447, 498 (2000) 
[4] A. M. Hoogenboom, Nucl. Instrum. Methods 3, 57 (1958)

[5] G. Rusev et al., Phys. Rev. C 77, 064321 (2008)

[6] A. Voinov et al., Phys. Rev. Lett. 93, 142504 (2004)

[7] A. C. Larsen et al., Phys. Rev. C 76, 044303 (2007)

[8] A. Bürger et al., Phys. Rev. C 85, 064328 (2012)

[9] A. C. Larsen et al., Phys. Rev. C 85, 014320 (2012)

[10] N. Syed et al., Phys. Rev. C 80, 044309 (2009)

[11] M. Guttormsen et al., Phys. Rev. C 83, 014312 (2011)

[12] A. C. Larsen et al., Phys. Rev. C 73, 064301 (2006)

[13] M. Guttormsen et al., Phys. Rev. C 71, 044307 (2005)

[14] M. Wiedeking et al., Phys. Rev. Lett. 108, 162503 (2012)

[15] E. Litvinova and N. Belov, Phys. Rev. C 88, 031302(R) (2013)

[16] R. Schwengner, S. Frauendorf, and A. C. Larsen, Phys. Rev. Lett. 111, 23504 (2013)

[17] B. Alex Brown and A. C. Larsen, Phys. Rev. Lett. 113, 252502 (2014)

[18] A. C. Larsen et al., Phys. Rev. Lett. 111, 242504 (2013)

[19] M. Guttormsen, A. Bürger, T. E. Hansen, and N. Lietaer, Nucl. Instrum. Methods Phys. Res. A 648, 168 (2011)

[20] M. Guttormsen et al., Phys. Scr., T 32, 54 (1990)

[21] M. Guttormsen, T. S. Tveter, L. Bergholt, F. Ingebretsen, and J. Rekstad, Nucl. Instrum. Methods Phys.
Res. A 374, 371 (1996)

[22] M. Guttormsen, T. Ramsøy, and J. Rekstad, Nucl. Instrum. Methods Phys. Res A 255 , 518 (1987).

[23] D. M. Brink, Ph.D. thesis, Oxford University, 1955.

[24] P. Axel, Phys. Rev. 126, 671 (1962).

[25] S. Amano et al., Nucl. Instrum. Methods in Phys. Res. A 602, 337 (2009)

[26] K. Horikawa, S. Miyamoto, S. Amano, and T. Mochizuki, Nucl. Instrum. Methods in Phys. Res. A 618, 209 (2010).

[27] B. L. Berman, S. C. Fultz, Rev. Med. Phys. 47, 713 (1975)

[28] T. Kondo et al., Nucl. Inst. and Meth. A 659, 462 (2011)

[29] D. M. Filipescu et al., Phys. Rev. C 90, 064616 (2014)

[30] H.-T. Nyhus et al., Phys. Rev. C 91, 015808 (2015)

[31] P. Carlos et al., Nuclear Physics A 258, 365 (1976)

[32] F. Bečvár̆, P. Cejnar, R. E. Chrien, and J. Kopecky, Phys. Rev. C 46, 1276 (1992)

[33] L. G. Moretto et al., arXiv:1406.2642 (2014)

[34] A. C. Larsen and S. Goriely, Phys. Rev. C 82, 014318 (2010)

[35] A. Koning, S. Hilaire and A. C. Duijvestijn, "TALYS-1.6", Proceedings of the International Conference on Nuclear Data for Science and Technology, April 22-27, 2007, Nice, France; EDP Sciences, 2008, p. $211-214$

[36] A. Spyrou et al., Phys. Rev. Lett. 113, 232502 (2014) 
